\title{
Correspondence
}

\section{Tendon rupture in systemic lupus erythematosus}

SIR, We note with interest the report on spontaneous rupture of weight bearing tendons in systemic lupus erythematosus (SLE). ${ }^{1}$ We report a case of rupture of a non-weight bearing tendon, namely the long head of biceps.

A 35 year old woman had a six year history of SLE, which has been clinically quiescent since 1982 . In January 1985 she sustained a fracture of the surgical neck of the left humerus with minimal displacement. This was treated conservatively, and she made an uneventful recovery. In August 1985 she presented with a one month history of spontaneous swelling in the left arm. Examination showed a non-tender mass in the middle third of the arm, which became more marked with resisted elbow flexion. Overall function was well preserved and surgical repair was not undertaken.

This is the first report of spontaneous rupture of a non-weight bearing tendon in SLE. At presentation, SLE was quiescent and there was no history of left shoulder arthritis or bicipital tenosynovitis. In keeping with previous reports ${ }^{1}$ our patient was on long term (four and a half years) corticosteroid therapy, possibly resulting in attenuation of the biceps tendon. It is unlikely that the humeral fracture caused the tendon rupture in January 1985 and remained undetected for seven months. We conclude that spontaneous rupture may occur in both non-weight bearing and weight bearing tendons in SLE patients.
Rheumatic Disease Unit,

Wellesley Hospital,

Toronto,

Canada

\section{Reference}

1 Potasman I, Bassan H M. Multiple tendon rupture in systemic lupus erythematosus: a case report and review of the literature. Ann Rheum Dis 1984; 43: 347-9. does not appear to have been established. It is important to know whether Muñoz-Gómez and colleagues had failed to show the presence of amyloid in the synovial fluid or synovium of asymptomatic patients on long term haemodialysis, in patients with primary hyperparathyroidism or, indeed, in age and sex matched controls. In this laboratory we have also been engaged in looking for amyloid in material removed at carpal tunnel decompression. While in our study biopsy specimens from patients receiving haemodialysis have shown amyloid deposits in perineural connective tissue, so too have specimens from other members of the 'control' group, all of whom have normal renal function.

Even if it could be shown that the amyloid deposition described by Muñoz-Gómez et al is unique to those patients on chronic haemodialysis who present with joint symptoms it still does not establish a causal relationship. It would be just as valid to claim that because most patients with osteoarthritis have amyloid within their joints, then amyloid is the cause of osteoarthritis.

The clinical and pathological findings of Muñoz-Gómez et al are interesting, but I can see nothing in the present paper which excludes their being epiphenomena. Until such time as properly controlled studies are performed and a mechanism linking the clinical syndrome with the microscopic findings can be identified there appears to be little justification for using the term 'amyloid arthropathy', with all its attendant pathogenetic implications, for the disorder which they describe.

University of Manchester, A J FREEMONT

Department of Rheumatology,

Stopford Building,

Oxford Road,

Manchester M13 9PT

\section{References}

1 Muñoz-Gómez J, Bergadá-Barado E, Gómez-Pérez, et al. Amyloid arthropathy in patients undergoing periodical haemodialysis for chronic renal failure: a new complication. Ann Rheum Dis 1985; 44: 729-33.

2 Rowe I F. Editorial: Amyloid arthropathy. Ann Rheum Dis 1985; 44: 727-8.

\section{Antikeratin antibody in rheumatoid and psoriatic arthritis}

SIR, I was interested in the recent paper by Muñoz-Gómez et al on amyloid arthropathy ${ }^{1}$ and Dr Rowe's very readable editorial review ${ }^{2}$ also entitled 'Amyloid arthropathy' which preceded it. I am, however, concerned by the name given to this disorder which implies a causative relation between amyloid in the joint and the patients' symptoms. Although it is undeniably possible that this could be the case, the link
SIR, We were interested to read the recently published studies $^{12}$ investigating the disease specificity of antikeratin antibodies. The knowledge that psoriasis is a disorder characterised by an abnormality of keratinisation prompted us to carry out a preliminary study into the prevalence of antikeratin antibodies in patients with 